\title{
Is Conservative Treatment with Antibiotics the Correct Strategy for Management of Right Colonic Diverticulitis?: A Prospective Study
}

\author{
Tae Jung Kim, In Kyu Lee, Jong Kyung Park, Yoon Suk Lee, Youn Si, Hun Jung, Hyung Jin Kim, \\ Sang Chul Lee, Dae Young Cheung ${ }^{1}$, D. Lee Gorden², Seung Taek Oh \\ Departments of Surgery and ${ }^{1}$ Internal Medicine, The Catholic University of Korea School of Medicine, Seoul, Korea; ${ }^{2}$ Department of Surgery, \\ Vanderbilt University Medical Center, Nashville, TN, USA
}

Purpose: The goals of this study were to identify whether conservative treatment with antibiotics in right colonic diverticulitis (RCD) patients, our empirical method used until now, is adequate and to determine how the natural history of RCD is affected by conservative treatment.

Methods: This study was designed as a case-control study. Group I was comprised of 12 patients who were managed conservatively, and clinical data were retrospectively collected. In group II, a total of 49 patients, diagnosed by using diagnostic criteria for RCD and managed conservatively, were prospectively included.

Results: The period of fasting was 2.7 days, and the hospital stay was 4.6 days in all patients. The intravenous and the oral antibiotic periods were 3.8 days and 9.8 days, respectively. There were no statistically significant differences in treatment results between the two groups except the duration of fasting and the hospitalization, and there were no complications under conservative treatment. Eight patients (13.1\%) had recurrent diverticulitis during the follow-up period. The recurrence risk showed no significant difference between the groups. The RCD-free period after management was 60.1 months, and patients with recurrent RCD were treated by conservative treatment or laparoscopic surgery.

Conclusion: Conservative treatment with antibiotics is the optimal treatment of choice for RCD and shows no increase in complications.

Keywords: Diverticulitis; Colon, Ascending; Medical management

\section{INTRODUCTION}

Right colonic diverticulitis (RCD) is an unusual disease in Western countries, but it exhibits higher incidence in Asian countries [1-4]. Treatment strategies for RCD have been difficult to define due to its low diagnostic accuracy [5]. Aggressive surgical treatment is advocated by some investigators decrease incidence of com-

Received: June 10, 2011 Accepted: August 16, 2011

Correspondence to: In Kyu Lee, M.D.

Department of Surgery, Yeouido St. Mary's Hospital, The Catholic University of Korea School of Medicine, 62 Yeouido-dong, Yeongdeungpo-gu, Seoul

150-713, Korea

Tel: +82-2-3779-2235, Fax: +82-2-786-0802

E-mail: cmcgslee@catholic.ac.kr

(C) 2011 The Korean Society of Coloproctology

This is an open-access article distributed under the terms of the Creative Commons Attribution NonCommercial License (http://creativecommons.org/licenses/by-nc/3.0) which permits unrestricted noncommercial use, distribution, and reproduction in any medium, provided the original work is properly cited. plications associated with conservative treatment [6-9]. However, recent reports have shown comparable results for conservative medical treatment of uncomplicated RCD, and the results are considered as acceptable because of the benign and self-limited natural history of RCD [5, 10-12].

A previous comparative study of treatment modalities, conservative antibiotics treatment, surgical treatment, and appendectomy, demonstrated no difference in the frequency of complications or the recurrence rates [5]. Moreover, most RCD patients had multiple diverticula in the colon. If it is diagnosed preoperatively, uncomplicated RCD can be managed conservatively with intravenous antibiotics. However, segmental resection is advocated as the most effective treatment when it is diagnosed intraoperatively. Surgical intervention is also beneficial in the aspect of recurrence. Previously, a set of diagnostic criteria for RCD that increased the preoperative diagnosis rate up to 85 percent was proposed, and that set was applied in this study [13], which was aimed to evaluate pro- 
spectively the clinical implications in RCD patients receiving conservative treatment with antibiotics.

\section{METHODS}

\section{Patients}

This study was designed as a case-control study to verify that there's no difference between the control group and the case (experimental) group. The flow chart of patient selection is presented below (Fig. 1).

The control group (group I) was comprised of 12 patients who were managed conservatively after making a diagnosis of RCD at the Department of Surgery, St. Mary's Hospital, The Catholic University of Korea School of Medicine, from January 2000 to December 2005. Clinical data for the control group were retrospectively collected based on medical records.

A total of 54 patients, diagnosed using the established diagnostic criteria for RCD [13], were enrolled as case group (group II) from January 2006 to 2007 and from September 2008 to 2009. Subsequently, a total of 49 patients were included, and clinical data for

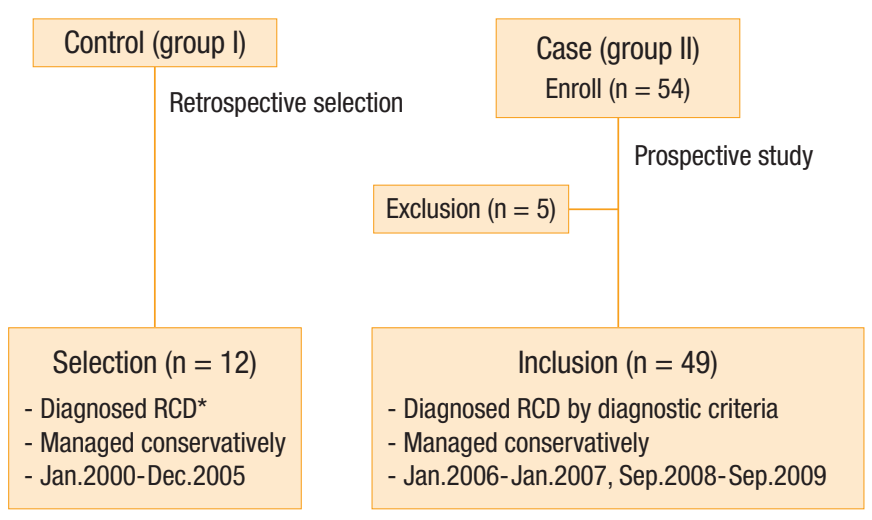

Fig. 1. The flow chart of patient selection. RCD, right colon diverticulitis. those were prospectively collected for analysis.

The median follow-up time was 15.1 months (range, 1 to 90 months). All patients included provided informed consent upon entering the study. This study was approved by the Institutional Review Board of Ethical Committee of the College of Medicine (SC10OESI0079).

\section{Diagnosis}

The RCD was confirmed by using abdominal ultrasound or computed tomography (CT) and barium enema (BE) in both groups. Abdominal ultrasound or CT was checked in patients complaining of symptoms at admission, and BE was performed in the outpatient clinic after discharge.

The diagnostic criteria for RDC were mentioned in Table 1 [13]. Each major criterion was given a score of two points; a minor criterion received one point. An abdominal CT scan was performed following informed consent if the total number of points exceeded two. Major diagnostic criteria included 1) non-migratory pain to the right lower quadrant; 2) a leukocyte count $<10,000 / \mathrm{mm}^{3} ; 3$ ) lateralized abdominal pain; and 4) a history of right colonic diverticulum diagnosed by using BE or colonoscopy. Minor diagnostic criteria included 1) a history of right lower quadrant abdominal pain, 2) no symptoms of nausea or vomiting, 3) symptoms of constipation or diarrhea, and 4) abdominal pain for at least seven days.

Recurrence of RCD was defined as a condition in which abdominal pain recurred and antibiotic therapy was needed based on radiologic findings after first management. Classification of a diverticular abscess was made as proposed by Hinchey [14].

\section{Treatment}

Conservative treatment for the patients with group I included 1) broad-spectrum antibiotics or 2) percutaneous drainage (PCD) in patients with a large abscess. The patients with group II received a combination therapy of second generation cephalosporin, aminoglycoside, and metronidazole during the hospitalization periods and oral antibiotics for 7 days after discharge. If abdominal tender-

Table 1. Diagnostic criteria for right colonic diverticulitis

\begin{tabular}{lll}
\hline Diagnostic criteria & & Positive \\
\hline Major (2 point) & No migration pain to the right lower quadrant \\
& Leukocyte count $<10,000 / \mathrm{mm}^{3}$ \\
& Lateralized abdominal pain \\
& History of right colonic diverticulum \\
& History of the same abdominal pain \\
Minor (1 point) & No nausea/vomiting symptoms \\
& History of diarrhea or constipation \\
& Abdominal pain for at least seven days \\
\hline Total points & \\
\hline
\end{tabular}

Subjects: adult patients with suspected acute appendicitis. Points $\geq 3$, perform abdominal computed tomography under the impression of diverticulitis; Points $<3$, no further study. 
ness persisted, patients took oral antibiotics for a longer period. Oral intake of foods was started if there was no abdominal pain even when abdominal tenderness was present.

\section{Statistical analysis}

The clinical characteristics were compared in both the two groups. The results for continuous variables are expressed as mean \pm standard deviation. A univariate statistical analysis was performed using the Mann-Whitney U-test for continuous independent samples and the chi-square or Fisher's exact test for categorical variables. Probabilities of recurrence were computed using the KaplanMeier method with the log-rank test. The statistical analyses were performed using SPSS ver. 12.0 (SPSS Inc., Chicago, IL, USA), and a P-value $<0.05$ was considered to indicate a statistically significant difference at the $95 \%$ confidence interval.

\section{RESULTS}

\section{Clinical characteristics}

The clinical characteristics of included patients are presented Table 2. The male-to-female ratio of the patients with RCD was 1.9:1, and the mean age was $37.4 \pm 1.1$ years. No migratory pain in right lower quadrant (RLQ) was observed in 41 patients (67.2\%) of the 61 patients in the two groups (12 in group I and 29 in group II),

Table 2. Comparison of clinical characteristics between the two groups

\begin{tabular}{|c|c|c|c|c|}
\hline & Total $(n=61)$ & Group I $(n=12)$ & Group II $(n=49)$ & P-value \\
\hline Age (yr) & $34.9 \pm 9.0$ & $36.9 \pm 11.2$ & $33.5 \pm 7.3$ & 0.363 \\
\hline Gender & & & & 0.518 \\
\hline Female & $21(34.4)$ & $3(25.0)$ & $18(36.7)$ & \\
\hline Male & $40(65.6)$ & $9(75.0)$ & $31(63.3)$ & \\
\hline Body temperature $\left({ }^{\circ} \mathrm{C}\right)$ & $37.3 \pm 0.6$ & $37.3 \pm 0.8$ & $37.4 \pm 0.4$ & 0.551 \\
\hline Initial leukocyte count (/mm³) & $11,516.0 \pm 2,980.0$ & $11,375.8 \pm 3,085.8$ & $11,609.4 \pm 2,994.1$ & 0.838 \\
\hline Diagnostic criteria $(n=61)$ & & & & 0.588 \\
\hline True positive & $55(90.2)$ & $12(100)$ & $43(87.8)$ & \\
\hline False negative & $6(9.8)$ & - & $6(12.2)$ & \\
\hline \multicolumn{5}{|l|}{ Major diagnostic criteria } \\
\hline No pain migration to RLQ & $41(67.2)$ & $12(100)$ & $29(59.2)$ & 0.007 \\
\hline Leukocyte count $<10,000 / \mathrm{mm}^{3}$ & $21(34.4)$ & $4(33.3)$ & $17(34.7)$ & 1.000 \\
\hline Lateralized abdominal pain & $25(41.0)$ & $4(33.3)$ & $21(42.9)$ & 0.745 \\
\hline History of RCD & $9(14.8)$ & $4(33.3)$ & $5(10.2)$ & 0.065 \\
\hline \multicolumn{5}{|l|}{ Minor diagnostic criteria } \\
\hline History of the same pain & $10(16.4)$ & $1(8.3)$ & $9(18.4)$ & 0.670 \\
\hline No N/N symptom & $43(70.5)$ & $10(83.3)$ & $33(67.3)$ & 0.481 \\
\hline History of $D / C$ & $14(23.0)$ & $2(16.7)$ & $12(24.5)$ & 0.440 \\
\hline Abdominal pain over 6 days & $3(4.9)$ & - & $3(6.1)$ & 1.000 \\
\hline \multicolumn{5}{|l|}{ Diagnostic radiologic tools } \\
\hline Ultrasonography (n=12) & & & & 1.000 \\
\hline True positive & $6(50.0)$ & $3(42.9)$ & $3(60.0)$ & \\
\hline False negative & $6(50.0)$ & $4(57.1)$ & $2(40.0)$ & \\
\hline Abdominal CT $(n=57)$ & & & & 1.000 \\
\hline True positive & $55(96.5)$ & $8(100)$ & $47(95.9)$ & \\
\hline False negative & $2(3.5)$ & - & $2(4.1)$ & \\
\hline Barium enema $(n=61)$ & & & & 0.203 \\
\hline Single diverticulum & $11(18.0)$ & $4(33.3)$ & $7(14.3)$ & \\
\hline Multiple diverticula & $50(82.0)$ & $8(66.7)$ & $42(85.7)$ & \\
\hline
\end{tabular}

Values are presented as mean $\pm \mathrm{SD}$ or number (\%).

RLQ, right lower quadrant; RCD, right colon diverticulum; N/N, nausea and vomiting. 
and the difference between the two groups was statistically significant $(\mathrm{P}=0.007)$. There were no differences between group I and group II except for the symptom of migratory pain.

Using diagnostic criteria, RCD could be diagnosed in 55 patients (90.2\%) of the 611 patients in the two groups. The mean scores for the diagnostic criteria were $4.3 \pm 1.7$ points.

\section{Diagnostic radiographic findings}

Ultrasound was performed in 12 patients and abdominal CT in 57 patients (Table 1). To confirm diverticulum, a BE was performed after $37.9 \pm 13.9$ days, and it presented multiple diverticula in 50 patients $(82.0 \%)$. The locations of the diverticula were confined to the cecum in nine patients (14.8\%) and to the ascending colon in twenty-one patients (34.4\%), were found in both the cecum and the ascending colon in twenty-two patients $(36.1 \%)$, ranged from the cecum to the transverse colon in four patients (6.6\%), and expanded throughout the entire colon in five patients (8.2\%). According to Hinchey's classification for complicated diverticulitis, only seven patients (11.5\%) were diagnosed as stage I.

\section{Clinical results of conservative treatment}

There were statistically significant differences between the two groups in duration of nil per os (NPO) and hospitalization, with the duration of NPO and the hospitalization being significantly shorter in group II $(\mathrm{P}<0.001)$ (Table 3$)$. In seven patients with complicated RCD, six received conservative medical treatment, and only one patient with a large abscess underwent CT-guided PCD. There were no serious complications following the procedure.

\section{Long-term outcomes after conservative treatment}

Eight patients (13.1\%) had recurrent diverticulitis during the follow-up period. However, there was no significant difference in recurrence between groups $(\mathrm{P}=0.650)$. The difference of recurrence risk between groups was also not statistically significant $(\mathrm{P}=0.602)$ (Fig. 2). The estimated RCD-free period after management was 60.1 months (limited to 90-month follow-up).

Among recurrent patients, six were managed by conservative treatment with bowel rest and intravenous antibiotics and were discharged without specific sequela. One patient underwent an

Table 3. Comparison of the clinical results of conservative medical treatment between groups

\begin{tabular}{lcccc}
\hline & Total $(\mathrm{n}=61)$ & Group I $(\mathrm{n}=12)$ & Group II ( $\mathrm{n}=49)$ & P-value \\
\hline Recovery time of normal temperature (day) & $0.7 \pm 0.9$ & $0.7 \pm 0.8$ & $0.7 \pm 0.9$ & 0.963 \\
Recovery time of normal leukocyte count (day) & $1.2 \pm 1.1$ & $1.4 \pm 1.4$ & $4.1 \pm 1.1$ & $3.7 \pm 1.0$ \\
Duration of IV antibiotics (day) & $3.8 \pm 1.1$ & $7.2 \pm 1.5$ & $10.4 \pm 4.5$ \\
Duration of oral antibiotics (day) & $9.8 \pm 4.9$ & $3.5 \pm 6.2$ & $2.4 \pm 1.0$ \\
Duration of fasting (day) & $2.7 \pm 1.1$ & $5.8 \pm 1.0$ & $4.3 \pm 1.1$ & 0.345 \\
Duration of hospitalization (day) & $4.6 \pm 1.3$ & $35.4 \pm 22.3$ & $37.0 \pm 9.6$ & $<0.001$ \\
Timing of BE examination (day) & $37.9 \pm 13.9$ & $2(16.7)$ & 6.001 \\
Recurrence (n, \%) & $8(13.1)$ & & 0.641 \\
\hline
\end{tabular}
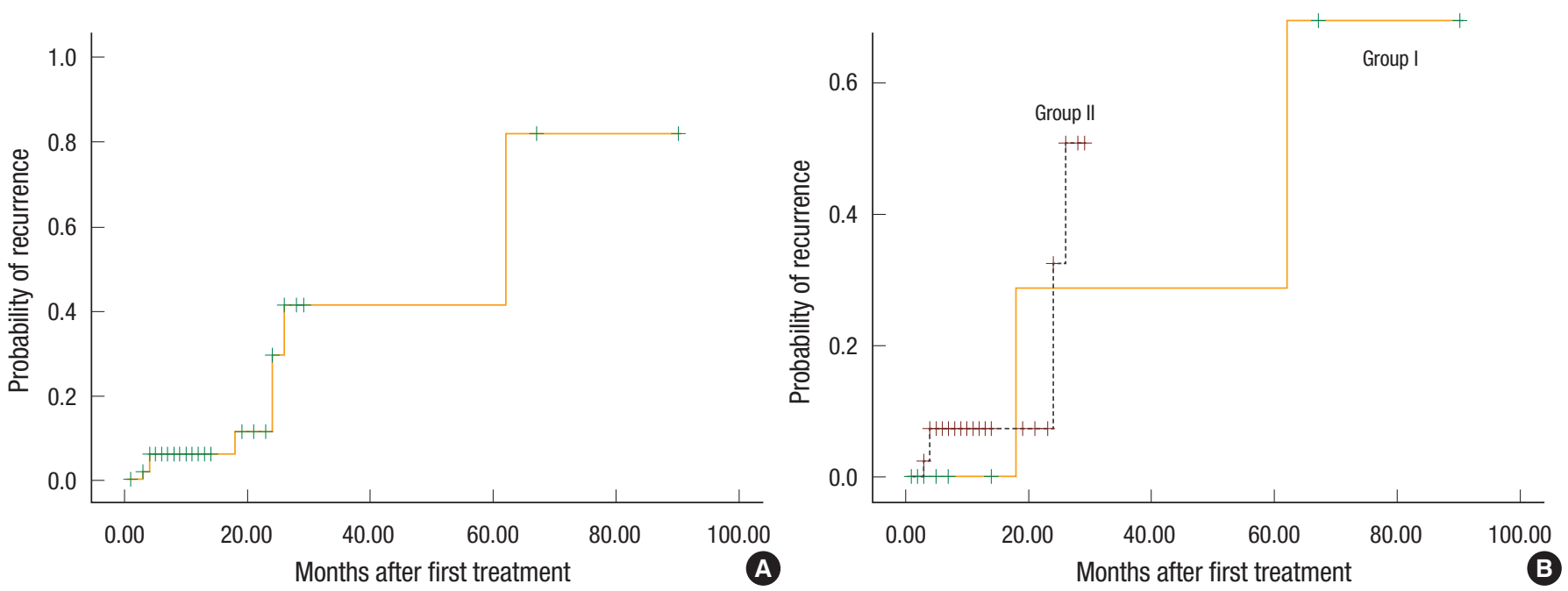

Fig. 2. Probability of recurrence after first treatment in all patients (A) and according to groups (B). The Kaplan-Meier estimated recurrence risk was not significantly different between the retrospective study group (group I) and prospective study group (group II) $(\mathrm{P}=0.602)$. 
emergency laparoscopic diverticulectomy because of a pericolic abscess at 18 months after the initial diagnosis, and another patient underwent a laparoscopic right hemicolectomy because of multiple diverticla. There was no morbidity or mortality.

\section{DISCUSSION}

The adequate treatment strategy for RCD has been controversial. Some studies have reported that conservative medical treatment would be complicated by high recurrence rates [6-9]. However, recent studies have reported that conservative medical treatment has acceptably low recurrence rates and is sufficient for treatment of recurrent RCD without complications and without the need for surgery [5, 10-12]. We previously reported that no differences in the rates of complications and recurrence existed between the conservative medical treatment and the surgical treatment groups. We also recommended adequate surgical correction in elective base after conservative medical treatment due to the high incidence of multiple diverticula [13]. Moreover, another study suggested that conservative medical therapy is a safe and effective treatment method [5], but these results were limited by the study being a retrospective analysis. Therefore, we performed this prospective study using a single arm setting.

In this study, all RCD (including complicated type I disease) patients were treated without specific sequela and revealed acceptably low recurrence rate $(16.7 \%)$ after conservative medical management. Recurrent patients could be treated by using conservative treatment, followed by staged surgery only in selected patients. Recurrent patients underwent laparoscopic surgery, with additional information regarding the locations and the number of right colonic diverticula.

In this study, no significant differences in any data except for the symptom of migratory pain into RLQ were observed. These results are consistent with previous results that conservative medical treatment for RCD was beneficial, and provide further evidence that earlier results were not due to selection bias and may be correct [5, 10-12].

In methods of conservative medical treatment, sequential therapy by using intravenous and oral antibiotics for at least 3 and 7 days, respectively, was reported to be consistent with the results of 2.0 days of bowel rest and 4.2 days of hospital stay [10]. In this study, the periods of NPO and hospital stay were 2.7 days and 4.6 days, respectively. If the time to recovery is considered, however, the periods of treatment and hospitalization are shortened; the normalizations of fever and leukocyte counts occurred after 0.7 and 1.2 days, respectively.

Conservative treatment usually ranges from 1-2 weeks in duration. Broad spectrum antibiotics have generally been recommended for use in the treatment of diverticular disease because the colonic flora is comprised of aerobes and anaerobes [15]. A variety of antibiotic regimens have been proposed. If symptoms are severe, however, the use of 1) a combination of ciprofloxacin and gentamicin or metronidazole; 2) amoxicillin/clavulanic acid; or 3) sulfamethoxazole/trimethoprim is recommended. Ampicillin, gentamicin, metronidazole, piperacillin, and tazobactam have also been successfully used in clinical practice for patients with severe and complicated diverticulitis whereas ciprofloxacin, metronidazole, and rifaximin have been successfully used in cases of uncomplicated diverticulitis [16]. Recently, based on the theory that some of the pathogenesis of colon diverticulitis are similar to those involved in inflammatory bowel disease, some studies have recommended the use of mesalazine in the treatment of diverticulitis [15].

This study establishes diagnostic criteria for RCD. As in a previous report, performing a CT scan after the application of the diagnostic criteria for RCD increased the accuracy of the preoperative RCD diagnosis [13]. This study demonstrated a preoperative diagnostic accuracy of $85.7 \%$. Use of these criteria allows for the application of principles derived from conservative medical management for sigmoid diverticulitis. In conclusion, the results of this study were similar to those from previous studies examining the management of RCD, and conservative treatment with antibiotics is the treatment of choice for RCD and can be used without increasing the complication rate.

\section{CONFLICT OF INTEREST}

No potential conflict of interest relevant to this article was reported.

\section{REFERENCES}

1. Schuler JG, Bayley J. Diverticulitis of the cecum. Surg Gynecol Obstet 1983;156:743-8.

2. Chia JG, Wilde CC, Ngoi SS, Goh PM, Ong CL. Trends of diverticular disease of the large bowel in a newly developed country. Dis Colon Rectum 1991;34:498-501.

3. Chan CC, Lo KK, Chung EC, Lo SS, Hon TY. Colonic diverticulosis in Hong Kong: distribution pattern and clinical significance. Clin Radiol 1998;53:842-4.

4. Yap I, Hoe J. A radiological survey of diverticulosis in Singapore. Singapore Med J 1991;32:218-20.

5. Lee IK, Kim SH, Lee YS, Kim HJ, Lee SK, Kang WK, et al. Diverticulitis of the right colon: tips for preoperative diagnosis and treatment strategy. J Korean Soc Coloproctol 2007;23:223-31.

6. Reid MR, Poer DH, Merrell P. A statistical study of 2,921 cases of appendicitis. JAMA 1936;106:665-9.

7. Fang JF, Chen RJ, Lin BC, Hsu YB, Kao JL, Chen MF. Aggressive resection is indicated for cecal diverticulitis. Am J Surg 2003;185: 135-40.

8. Ngoi SS, Chia J, Goh MY, Sim E, Rauff A. Surgical management of right colon diverticulitis. Dis Colon Rectum 1992;35:799-802.

9. Harada RN, Whelan TJ Jr. Surgical management of cecal diverticulitis. Am J Surg 1993;166:666-9.

10. Moon HJ, Park JK, Lee JI, Lee JH, Shin HJ, Kim WS, et al. Conservative treatment for patients with acute right colonic diverticulitis. 
Am Surg 2007;73:1237-41.

11. Lo CY, Chu KW. Acute diverticulitis of the right colon. Am J Surg 1996;171:244-6.

12. Oudenhoven LF, Koumans RK, Puylaert JB. Right colonic diverticulitis: US and CT findings--new insights about frequency and natural history. Radiology 1998;208:611-8.

13. Lee IK, Jung SE, Gorden DL, Lee YS, Jung DY, Oh ST, et al. The diagnostic criteria for right colonic diverticulitis: prospective eval- uation of 100 patients. Int J Colorectal Dis 2008;23:1151-7.

14. Hinchey EJ, Schaal PG, Richards GK. Treatment of perforated diverticular disease of the colon. Adv Surg 1978;12:85-109.

15. Floch MH, White JA. Management of diverticular disease is changing. World J Gastroenterol 2006;12:3225-8.

16. Tursi A. Acute diverticulitis of the colon--current medical therapeutic management. Expert Opin Pharmacother 2004;5:55-9. 